\title{
Anaesthetic management of a case of alkaptonuria for total knee arthroplasty
}

\author{
*A Ardhanari, ${ }^{1}$ VKV Seetharam ${ }^{2}$, MT Subbaramaiah $^{2}$, MS Kumaraswamy $^{3}$ \\ Senior Registrar ${ }^{1}$, Consultant ${ }^{2}$, Senior Consultant ${ }^{3}$ \\ Department of Anaesthesiology and Pain Relief, Apollo Specialty Hospital, Bengaluru, \\ Karnataka, India.
}

*Corresponding author:dr.anbarasan82@gmail.com

\begin{abstract}
Alkaptonuria, is a rare autosomal recessive disorder of tyrosine metabolism caused by a deficiency of homogentisate 1,2dioxygenase. It results in accumulation of homogentisic acid in spine and large joints and cardiac valves. It also involves respiratory, airway, genitourinary, cutaneous and ocular systems. Administering spinal anaesthesia is extremely challenging in these cases. We report a case of a $66 \mathrm{yr}$ old male alkaptonuric ochronosis with symptoms of ankylosing spondylitis, hypertension and obstructive sleep apnoea for total knee replacement successfully managed intraoperatively with subarachnoid block after marking the interspinous space with ultrasound.
\end{abstract}

Keywords: Alkaptonuria; ochronosis; anaesthesia; ultrasound

\section{Introduction}

Alkaptonuria is an autosomal recessive genetic disorder of tyrosine metabolism characterized by the triad of homogentisic aciduria, ochronosis, and arthritis. ${ }^{1}$ The condition affects one in 250,000 to one million people worldwide. ${ }^{2}$ The clinical manifestations include a history of urine turning dark on standing and on alkalization due to elimination of excessive amounts of homogentisic acid (HGA). Other manifestations are ochronosis, a blue-black pigmentation of connective tissues and cartilages; and arthritis of weight bearing joints. ${ }^{3-5}$ The most common clinical manifestations of ochronosis involve the musculoskeletal, respiratory, airway, cardiovascular, genitourinary, cutaneous, and ocular systems. We report the successful perioperative anaesthetic management of an alkaptonuric patient with comorbidities.

\section{Case Report}

A 66 year old, $80 \mathrm{~kg}$ gentleman, with complaints of pain of both knees, was scheduled for right total knee replacement. His medical history revealed alkaptonuria, hypertension, obstructive sleep apnoea and daytime somnolence. He had been diagnosed to have alkaptonuria 46 years back. Medical therapy included telmisartan 40mg once a day for hypertension and oral NSAID on and off for knee pain.
There was no previous history of any surgery.

On examination, patient was conscious, oriented and afebrile. His pulse rate was 64 per $\mathrm{min}$ and blood pressure of 130/90mmHg. Airway examination revealed an adequate mouth opening, thyromental distance of $6 \mathrm{~cm}$, restricted extension of neck and Mallampati class III. Cardiovascular examination was unremarkable. Examination of respiratory system revealed occasional rhonchi.

Investigations including haematological (haemoglobin, $13.9 \mathrm{~g} / \mathrm{dL}$ ), renal, liver functions, thyroid function, and coagulation profile were normal. Chest x-ray demonstrated cardiomegaly and the 12 lead ECG showed left ventricular hypertrophy $(\mathrm{LVH})$. Echocardiogram revealed a left ventricular ejection fraction of $58 \%$ without any regional wall motion anomalies, concentric LVH, mild pulmonary hypertension and left ventricular dysfunction.

The patient was accepted for right Total knee replacement (TKA) under ASA functional class 3, explaining the high perioperative risk due to multiple comorbid illnesses. He was advised to stop telmisartan on the day of surgery. Preoperative incentive 
spirometry and levosalbutamol nebulisation were also advised. Regional anaesthesia with subarachnoid block and femoral nerve nerve block with catheter insertion and single shot sciatic nerve block was planned.

In the operating theatre, an $18 \mathrm{G}$ intravenous line was secured after application of ECG, pulse oximeter $\left(\mathrm{SpO}_{2}\right)$, and non-invasive blood pressure (NIBP) monitors. Before administering spinal anaesthesia, L3-L4 interspinous space was marked with the help of ultrasound as difficulty was anticipated due to his pre-existing symptoms of ankylosing spondylitis and ochronosis. After local infiltration of $2 \%$ lignocaine, subarachnoid block was successfully administered in a single attempt with a $23 \mathrm{G}$ spinal needle. $0.5 \%$ levobupivacaine $3 \mathrm{ml}$ was injected after aspiration for free flow of clear CSF was confirmed. Level of block up to T10 was achieved. Ultrasound combined with peripheral nerve stimulation (PNS) guided femoral nerve block was given with $0.25 \%$ levobupivacaine $20 \mathrm{ml}$ and dexamethasone $8 \mathrm{mg}$. A catheter was inserted at this site. Single shot sciatic nerve block with $0.2 \%$ ropivacaine $20 \mathrm{ml}$ was administered successfully. The surgery lasted for $2 \mathrm{~h}$ and vitals were stable throughout.1.5L of lactated ringer solution was infused. There was minimal blood loss as a tourniquet was used. Intraoperative urine output was $400 \mathrm{ml}$. At the end of the procedure, patient was shifted to intensive care unit for observation. Postoperative haemoglobin was $11 \mathrm{~g} / \mathrm{dl}$. Intravenous paracetamol $1 \mathrm{~g}$ thrice a day was prescribed for pain control along with $0.2 \%$ ropivacaine at $5 \mathrm{ml} / \mathrm{hr}$ through continuous femoral nerve block catheter. NSAID was avoided. Next day, the patient was shifted to the ward and advised regular physiotherapy with followup in the orthopaedic clinics.

\section{Discussion}

Alkaptonuria is a rare inherited genetic disorder of tyrosine metabolism characterized by the triad of homogentisic aciduria, ochronosis, and arthritis. ${ }^{1,6}$ A toxic tyrosine by product HGA accumulates in blood and is excreted in urine in large amounts due to a lack of enzyme homogentisic acid oxidase. Ochronosis results from the deposition of this brown- coloured pigment in the cartilaginous and musculoskeletal system in the body.

The presence of alkaptonuria with difficult airway and obstructive sleep apnoea made the perioperative management of our patient challenging. Care of a patient with alkaptonuric ochronosis is complex and challenging. There are no formal guidelines for the perioperative management of the patient with ochronosis. Organ and tissue involvement will influence the anaesthetic technique. ${ }^{2}$ A thorough evaluation of the type and severity of systemic dysfunction is essential before administration of anaesthesia.

The depositions of pigments make the cartilage of the joints prone to destruction and make the joints painful. The cartilage of the airway and respiratory system too may be affected in ochronosis. ${ }^{1}$ Heavy deposition of the pigment in the laryngeal, tracheal, and bronchial cartilages may result in hoarseness, dysphagia, and a difficult airway.

Degenerative changes occur in spine and vertebral discs. ${ }^{7}$ Onchronosis of spine results in narrowing of interspinous spaces making spinal anaesthesia challenging as in our case. Use of ultrasound to mark the interspinous space proved to be valuable in our case as opposed to use of anatomical landmarks.

Cardiovascular abnormalities such as calcified and stenotic valves, generalized atherosclerosis, and conduction blocks may also be associated with ochronosis. ${ }^{1}$ A thorough preoperative evaluation of the cardiovascular status is essential. The accumulation of HGA predisposes the patient to the formation of renal calculi which in turn can be responsible for frequent urinary tract infections, obstruction, and potentially failure. Our patient did not have any clinical symptoms related to renal dysfunction. Abnormalities in renal tubular function may result in inadequate excretion of HGA leading to a higher plasma level and thus increased deposition in tissues. ${ }^{5}$ This may lead to increased severity of various system dysfunctions. Associated comorbidities such as hypertension, as seen in our patient, too increase the chance of 
renal compromise in the perioperative period. Inability to monitor regional oxygen saturation, due to pigment deposition, has been reported in a patient with alkaptonuria. ${ }^{3}$ Caution should be exercised in pulse oximeter monitoring of patients with excessive pigment deposition.

\section{Conclusion}

Alkaptonuric patients can be managed successfully with regional anaesthesia when through preoperative evaluation is done and by use of ultrasound to identify interspinous spaces.

\section{References}

1. Collins E, Hand. Alkaptonuric ochronosis: A case report. AANA 2005;73:41-6

2. Fisher AA, Davis MW. Alkaptonuric ochronosis with aortic valve and joint replacements and femoral fracture. Clin Med Res2004;2:209-15

http://dx.doi.org/10.3121/cmr.2.4.209

3. Argiriadou $\mathrm{H}$, Anastasiadis $\mathrm{K}$, Antonitsis $\mathrm{P}$ et al. The inability of regional oxygen saturation monitoring in a patient with alkaptonuria undergoing aortic valve replacement. J Cardiothoracic VascAnest. 2009;23:586-8

http://dx.doi.org/10.1053/j.jvca.2008.08.014 PMid:18948036

4. Dom K, Pittevils T. Ochronoticarthroplasty: The black hip. Case report and review of literature. ActaOrthopBelg 1997;63:122-5 PMid:9265798
5. Introne WJ, Phornphutkul C, Bernardini I et al. Exacerbation of the ochronosis of alkaptonuria due to renal insufficiency and improvement after renal transplantation. $\mathrm{Mol}$ Genet Metab 2002;77:136-42 http://dx.doi.org/10.1016/S10967192(02)00121-X

6. Pandey R, Kumar A, Garg R et al. Perioperative management of patient with alkaptonuria and associated multiple comorbidities. J Anaesthesiol Clin Pharmacol. 2011; 27(2): 259-261 http://dx.doi.org/10.4103/0970-9185.81839 PMid:21772695 PMCid:PMC3127314

7. Ogata J, Tamura K, Miyanishi K et al. Anesthesia in a patient with alkaptonuricochronosis for total hip arthroplasty. Masui 2008;57:439-42 PMid:18416199 